\title{
Hand Posture Estimation in Complex Backgrounds by Considering Mis-match of Model
}

\author{
Akihiro Imai ${ }^{1}$, Nobutaka Shimada ${ }^{2}$, and Yoshiaki Shirai ${ }^{2}$ \\ ${ }^{1}$ Dept.of Computer-Controlled Mechanical Systems, \\ Osaka University Yamadaoka, Suita, Osaka 565-0871, Japan \\ 2 Dept.of Human and Computer Intelligence, Ritumeikan University, \\ Nojihigashi, Kusatsu, Shiga 525-8577, Japan
}

\begin{abstract}
This paper proposes a novel method of estimating 3-D hand posture from images observed in complex backgrounds. Conventional methods often cause mistakes by mis-matches of local image features. Our method considers possibility of the mis-match between each posture model appearance and the other model appearances in a Baysian stochastic estimation form by introducing a novel likelihood concept "Mistakenly Matching Likelihood (MML)“. The correct posture model is discriminated from mis-matches by MML-based posture candidate evaluation. The method is applied to hand tracking problem in complex backgrounds and its effectiveness is shown.
\end{abstract}

\section{Introduction}

Precise hand-finger shape estimation methods using visual cues have been developed [1] 2] [3] [4] [5] 6] in order to implement the gestural interfaces in a touch-less manner which are utilized in interaction with virtual environments and automatic sign-language translation.

One of the difficulties of implementing the interfaces based on the hand shape estimation exists in its situations where the interfaces are needed: its complex backgrounds like colorful and textured clothes, skin-colored region as human face and some desktops on which various tools and objects are scattered. Since the hand shape even in simple backgrounds is a tough problem due to its great varieties of posture, shape estimation with simultaneous segmentation is still a challenging problem.

To solve the problem with feasible computing resources, some trials were reported from the following two viewpoints: 1) how to reduce the number of posture candidates to consider (i.e. how to predict the posture), 2) how to evaluate the matching degree between the posture candidate and observed image features.

From the viewpoint 1), Active Shape Model [7] is proposed, which learns acceptable shape deformations and tracks the region contour or texture assuming smooth deformation and motion. Non-smooth deformation can be treated by introducing Switching Linear Dynamics [8]. 3-D model-based shape prediction and tracking, not based on appearance learning, is also proposed [5. Most of 


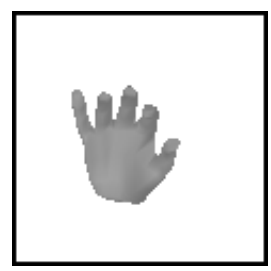

(a) Estimation result

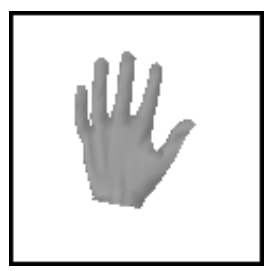

(c) Shape similar to input image

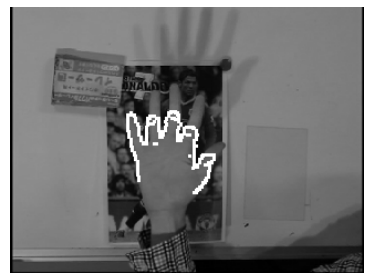

(b) Edges of estimation result put on input image

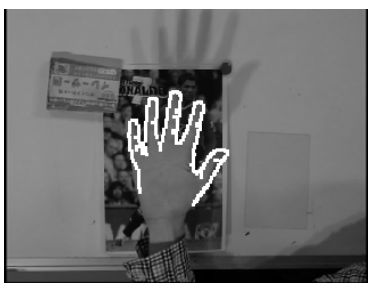

(d) Edges of similar shape put on input image

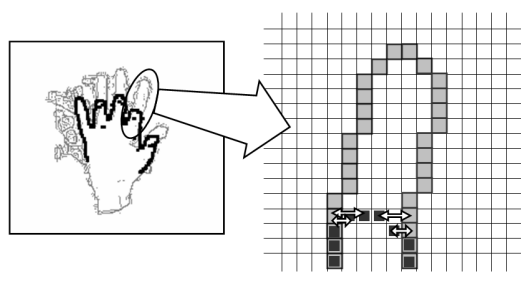

Fig. 2. Corresponding of edges

Fig. 1. Mistake of conventional method

those methods employ a parallel search scheme in tracking, like beam-search or particle filter for robustness against temporal mis-estimation and tracking failure 5] 9 [10 $11,12,13,14$.

While many improvements from the first viewpoint are reported, those from the second viewpoint, concerned with the evaluation of matching degree, are comparatively few and most implementations employ a simple feature corresponding and evaluating method: chamfer matching [15] 16. Chamfer matching makes correspondences between the features with the least distance in the image, and evaluates the matching degree by the sum of the distances (chamfer distance). This simple matching scheme, of course, causes often a wrong shape estimate on the complicated backgrounds.

Fig. 1 is an example of wrong estimate of hand posture caused by chamfer matching. Because many edge textures are observed in the hand region, the finger tips of the posture model are mistakenly corresponding to the inner edges (see Fig. 2) and as a result its chamfer distance is evaluated too small. While this problem is hard to avoid as long as using chamfer matching, no more appropriate matching method is found other than chamfer matching. Therefore the matching degree should be evaluated under the consideration of that such a wrong matching often happens.

Nevertheless, the existence of the mismatch caused by chamfer matching does not directly mean its uselessness. Embedding approach [15] evaluates the matching degree between an input image feature and not only one posture model but also several other reference models. For example of Fig. 1, in addition to the correct posture candidate (c), the candidate (a) also has so high matching 
degree that (a) is picked up as the estimate. However, if only (a) has the high matching degree when (a) should be the correct matching, these two cases can be discriminated by evaluating the matching degrees with both reference models (a) and (c). Since Embedding approach only uses an ad-hoc way by evaluating the squared sum of the matching degrees of all reference models, its estimate is not the optimum in Bayesian point of view.

This paper mathematically derives the Baysian form of Embedding approach. In its derivation, a novel concept of likelihood is introduced: Mistakenly Matching Likelihood ( $M M L)$, which predicts the high evaluation caused by wrong matching and gives the ability to discriminate the true estimate from the false match in a stochastic way. The derived MML-based candidate evaluation is applied to hand tracking problem in complex backgrounds and its effectiveness is experimentally shown.

\section{Acquisition of Typical Hand Posture Images}

3-D Hand model used in our research is originally wireframe model. The model is modified into a shaded model. The joint bending angles are denoted by $\theta_{b, t, 1}, \theta_{b, t, 2}, \theta_{b, t, 3}, \theta_{b, i, 1}, \theta_{b, i, 2}, \theta_{b, i, 3}, \ldots$ and opening angles at a base joint of the fingers are denoted by $\theta_{o, t}, \theta_{o, i}, \ldots$ (shown in Fig 3 ). The posture of the whole hand model is represented by translation $t_{x}, t_{y}, t_{z}$ and Ritalin $\theta_{r, x}, \theta_{r, y}, \theta_{r, z}$. As a whole, the shape of the hand model has 26 degrees of freedom

$$
\boldsymbol{\theta}=\left(\theta_{b, t, 1}, \ldots, \theta_{r, z}\right) \text {. }
$$

CG images of typical hand models are shown in Fig. 4. The finger joints dependently moves in natural actions 5. In index, middle, ring, pinky fingers, adjacent joint angles are usually similar. This kind of joint constraints reduce

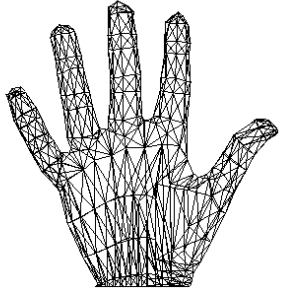

(a) Wireframe model

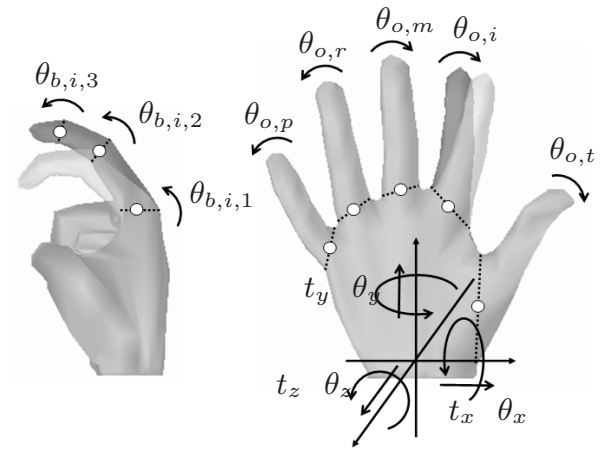

(b) Model after shading

Fig. 3. Hand model 


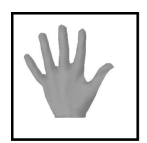

(a)

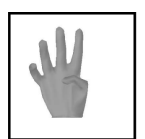

(b)

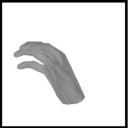

(c)

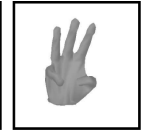

(d)

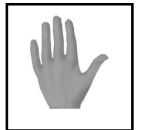

(e)
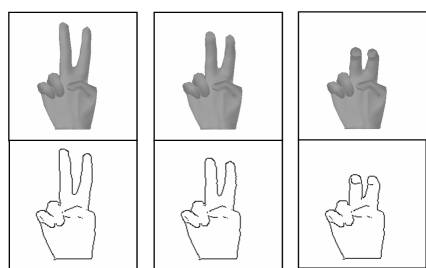

Fig. 4. CG images of typical hand postures

Fig. 5. Edge images of hand model

Table 1. Quantization width from search center

\begin{tabular}{|c|c|c|c|}
\hline$\Delta \theta_{\text {bend }}^{c}\left[{ }^{\circ}\right]$ & $-\zeta$ & 0 & $\zeta$ \\
\hline$\Delta \theta_{\text {open }}^{c}\left[^{\circ}\right]$ & -6 & 0 & 6 \\
\hline$\Delta \theta_{o, m}^{c}\left[^{\circ}\right]$ & - & 0 & - \\
\hline$\Delta \theta_{r, x}^{c}\left[^{\circ}\right]$ & -30 & 0 & 30 \\
\hline$\Delta \theta_{r, y}^{c}\left[^{\circ}\right]$ & -15 & 0 & 15 \\
\hline$\Delta \theta_{r, z}^{c}\left[^{\circ}\right]$ & -6 & 0 & 6 \\
\hline$\Delta t_{x}^{c}, \Delta t_{y}^{c}[\mathrm{~mm}]$ & -8 & 0 & 8 \\
\hline$\Delta t_{z}^{c}[m m]$ & -40 & 0 & 40 \\
\hline
\end{tabular}

the number of possible postures. Fig 5 shows the edge images generated from the $\mathrm{CG}$ images of the typical hand postures under the constraints.

The posture parameters $\theta$ to be estimated are quantized. The change is shown in Table 1 (The change of $\theta_{b}$ is represented by $\Delta \theta_{b e n d}^{c}$, that of $\theta_{o}$ other than middle is represented by $\Delta \theta_{\text {open }}^{c}$ ). Each parameter of $\theta_{b}$ is assumed to change 0 or $\pm \zeta$. Each parameter has own $\zeta$ between $9 \mathrm{deg}$ to $15 \mathrm{deg}$.

\section{Matching Method}

The system has several hand models with various dimensions: i.e. lengths and widths of the palm and fingers. Since input image sequences are assumed to start from a predefined simple shape and an initial position, the dimensions are easily initialized at the first frame. The posture parameters to be estimated are around the posture estimate at the previous image frame.

When each input image is obtained, the best-matched model is determined by the maximum likelihood criterion. Let $I$ denote edges and skin regions extracted from an input image and $\Theta_{j}$ denote a quantized parameter vector of the $j$ th model. The criterion is

$$
\hat{\Theta}=\arg \max _{\Theta_{j}} P\left(I \mid \Theta_{j}\right)
$$

where $P\left(I \mid \Theta_{j}\right)$ denotes the likelihood of the $j$ th model for the input. The likelihood is defined based on the difference of the image $I$ and the the appearance of the shape model. 


\subsection{Difference of Image and Appearance of Shape Model}

In this paper, edge image $I^{(e)}$ and skin-color region image $I^{(s)}$ are used as the image features of the input $I$. The difference between the silhouette of a typical hand model and that of an image is computed. Let $A_{\boldsymbol{\theta}}^{(s)}$ be the silhouette generated from a typical hand model $\boldsymbol{\theta}$. The Difference of the silhouette, $f_{\text {skin }}\left(A_{\boldsymbol{\theta}}^{(s)} ; I^{(s)}\right)$, is defined as the area of $A_{\boldsymbol{\theta}}^{(s)}$ which does not overlap with $I^{(s)}$.

The difference between the $I^{(e)}$ and the edge appearance $A_{\boldsymbol{\theta}}^{(e)}, f_{\text {dist }}\left(A_{\boldsymbol{\theta}}^{(e)} ; I^{(e)}\right)$, is computed by a modified chamfer matching, in which the edge points are classified by gradient direction and the edges is matched by the original chamfer matching in each direction class 13 18. The distance is weighted by the edge contrast and as a result $f_{\text {dist }}$ is defined as follows:

$$
f_{\text {dist }}\left(A_{\boldsymbol{\theta}}^{(e)} ; I^{(e)}\right)=\sum_{j} w_{\boldsymbol{\theta}, j} \min _{k}\left(\left\|\boldsymbol{x}_{\boldsymbol{\theta}, j}-\boldsymbol{x}_{I^{(e)}, k}\right\|+f_{I^{(e)}, k}+g(j, k)\right)
$$

where $\boldsymbol{x}_{\boldsymbol{\theta}, j}$ and $\boldsymbol{x}_{I^{(e), k}}$ denotes the $j$ th edge point of the model and the $k$ th edge point of an input edge image. $\|\cdot\|$ is 2 -dimensional Euclidean norm, $w_{\boldsymbol{\theta}, j}$ is a weight constant, $f_{I^{(e)}, k}$ is a penalty for an edge with a low contrast,

$$
\begin{gathered}
w_{\boldsymbol{\theta}, j}=\frac{d_{\boldsymbol{\theta}, j}}{\sum_{l} d_{\boldsymbol{\theta}, l}} . \\
f_{I^{(e),}, k}=-w_{d} d_{I^{(e)}, k}
\end{gathered}
$$

where $w_{d}$ represents weight constant. The difference of gradient direction $g(j, k)$ is defined in terms of gradient direction $\phi_{\boldsymbol{\theta}, j}$ of the model edge and gradient direction $\phi_{I^{(e)}, k}$ as

$$
g(j, k)=w_{\phi}\left\|\phi_{\boldsymbol{\theta}, j}-\phi_{I^{(e)}, k}\right\| .
$$

where $w_{\phi}$ represents weight constant. All weights are experimentally determined. The modified chamfer matching is computed by using distance transformation as fast as the original chamfer matching.

\subsection{Discrimination Principle}

The example of mis-matching by a conventional method has been shown as Fig. 1] in section 1. Let $\Theta_{a}$ and $\Theta_{c}$ respectively denote the posture parameter of model (a) (fist shape) and (c) (flat shape). Suppose one case that the true hand posture should be $\Theta_{c}$. In this section, we describe the principle to discriminate the true match from the wrong matches due to the complicated skin textures and the backgrounds and finally introduce its stochastic forms giving the discriminating criterion.

Then the probability of that the appearance $A_{\Theta_{c}}$ is matched to the input image, $p\left(A_{\Theta_{c}} \mid \Theta_{c}\right)$, should be large enough because the true posture is the same as the one which generates the appearance, $\Theta_{c}$.

On the other hand, the probability of $A_{\Theta_{a}}$ (small fist shape), $p\left(A_{\Theta_{a}} \mid \Theta_{c}\right)$ can be also large in spite of the posture difference between $\Theta_{a}$ and $\Theta_{c}$ because 


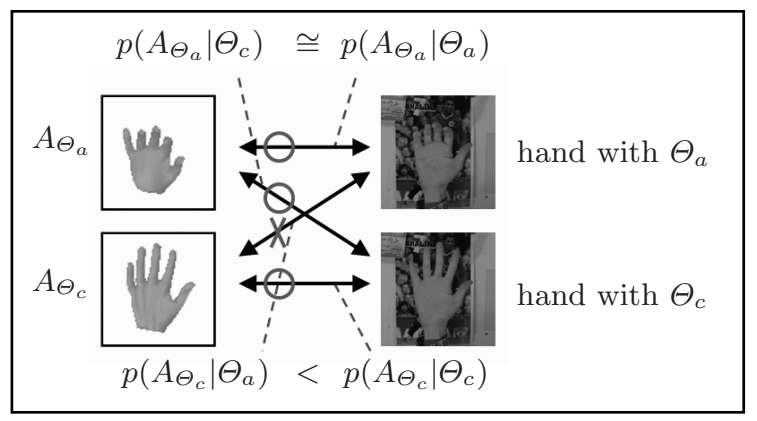

Fig. 6. Likelihood for edge images

almost all of the area of $A_{\Theta_{a}}$ is included and the inner texture edges can be wrongly matched to the finger contours. Therefore the conventional likelihood maximization can often choose $\Theta_{a}$ mistakenly for flat hand shapes like $\Theta_{c}$ due to the image capture noise, inaccuracy of the $3-\mathrm{D}$ shape model and quantization errors of the posture parameters.

In order to solve the mis-matches, we carefully analyze the behaviours of two more probabilities: $p\left(A_{\Theta_{a}} \mid \Theta_{a}\right)$ and $p\left(A_{\Theta_{c}} \mid \Theta_{a}\right) \cdot p\left(A_{\Theta_{a}} \mid \Theta_{a}\right)$ should be large and $p\left(A_{\Theta_{c}} \mid \Theta_{a}\right)$ should be small because $A_{\Theta_{c}}$ protrudes from the area of $\Theta_{a}$. The four probabilities take the behaviours as follows: while $p\left(A_{\Theta_{a}} \mid \Theta_{c}\right), p\left(A_{\Theta_{c}} \mid \Theta_{c}\right)$ are large together for the posture $\Theta_{c}, p\left(A_{\Theta_{a}} \mid \Theta_{a}\right)$ is large and $p\left(A_{\Theta_{c}} \mid \Theta_{a}\right)$ is small for the posture $\Theta_{a}$ (see Fig. 6). Therefore when the appearance $A_{\Theta_{a}}$ and $A_{\Theta_{c}}$ are observed together, that posture should be estimated as $\Theta_{c}$. When $A_{\Theta_{a}}$ is observed alone, that posture should be $\Theta_{a}$.

When the likelihood of an appearance $A_{\Theta_{k}}$ to a model $\Theta_{j}, p\left(A_{\Theta_{k}} \mid \Theta_{j}\right)$, is obtained for each possible combination of $k$ and $j$ in advance, the appropriate model can be chosen by taking the all $p\left(A_{\Theta_{k}} \mid \Theta_{j}\right)$ values into account like the above discussion.

When $k$ and $j$ are identical, $p\left(A_{\Theta_{k}} \mid \Theta_{j}\right)$ is equivalent to the conventional likelihood function. Otherwise, it means the likelihood of that an appearance $A_{\Theta_{k}}$ comes from a mistakenly chosen model $\Theta_{j}$. We call the likelihood as "mistakenly matching likelihood" (MML).

\subsection{Model Selection using Mistakenly Matching Likelihood}

We introduce the stochastic discrimination criterion from the principle described in the previous section by employing Bayesian estimation framework. Let $A_{\Theta_{1}}$, $A_{\Theta_{2}}, \ldots$ denote appearances of typical hand models. Assuming $A_{\Theta_{1}}, A_{\Theta_{2}}, \ldots$ are exclusive under each $\Theta_{j}$, the likelihood of $\Theta_{j}$ for $I$ can be expanded as below:

$$
p\left(I \mid \Theta_{j}\right)=\sum_{k} p\left(I, A_{\Theta_{k}} \mid \Theta_{j}\right)=\sum_{k} p\left(I \mid A_{\Theta_{k}}, \Theta_{j}\right) p\left(A_{\Theta_{k}} \mid \Theta_{j}\right) .
$$


Assuming the appearance $A_{\Theta_{k}}$ has all information to generate the observed image $I$, condition $\Theta_{j}$ can be removed,

$$
p\left(I \mid \Theta_{j}\right)=\sum_{k} p\left(I \mid A_{\Theta_{k}}\right) p\left(A_{\Theta_{k}} \mid \Theta_{j}\right) .
$$

In the conventional maximum likelihood estimation method, the likelihood for the case of $k=j$ is considered alone. In contrast, we additionally consider the MML for the case of $k \neq j$.

Assuming that $I^{(e)}$ and $I^{(s)}$ are independent when a certain $\Theta_{j}$ is specified,

$$
p\left(I \mid \Theta_{j}\right)=p\left(I^{(e)} \mid \Theta_{j}\right) p\left(I^{(s)} \mid \Theta_{j}\right)
$$

is derived as the discrimination criterion in stochastic form.

The likelihood $p\left(I^{(e)} \mid \Theta_{j}\right)$ and $p\left(I^{(s)} \mid \Theta_{j}\right)$ are respectively derived from the following equations:

$$
\begin{aligned}
& p\left(I^{(e)} \mid \Theta_{j}\right)=\sum_{k} p\left(I^{(e)} \mid A_{\Theta_{k}}^{(e)}\right) p\left(A_{\Theta_{k}}^{(e)} \mid \Theta_{j}\right) \\
& p\left(I^{(s)} \mid \Theta_{j}\right)=\sum_{k} p\left(I^{(s)} \mid A_{\Theta_{k}}^{(s)}\right) p\left(A_{\Theta_{k}}^{(s)} \mid \Theta_{j}\right)
\end{aligned}
$$

The probabilistic distributions $p\left(A_{\Theta_{k}}^{(e)} \mid \Theta_{j}\right)$ and $p\left(I^{(e)} \mid A_{\Theta_{k}}^{(e)}\right)$ for edge images is introduced the following sections. Those for skin color silhouette, $p\left(A_{\Theta_{k}}^{(s)} \mid \Theta_{j}\right)$ and $p\left(I^{(s)} \mid A_{\Theta_{k}}^{(s)}\right)$ can be introduced in the same manner of those for edge image.

\subsection{Likelihood of Typical Hand Models for Appearances}

The likelihood of typical hand model is obtained as the following form because of quantization errors of $\Theta_{j}$.

$$
\begin{aligned}
p\left(A_{\Theta_{k}}^{(e)} \mid \Theta_{j}\right) & =\int_{\Theta_{j}} p\left(A_{\Theta_{k}}^{(e)}, \boldsymbol{\theta}_{\boldsymbol{j}} \mid \Theta_{j}\right) d \boldsymbol{\theta}_{\boldsymbol{j}} \\
& =\int_{\Theta_{j}} p\left(A_{\Theta_{k}}^{(e)} \mid \boldsymbol{\theta}_{\boldsymbol{j}}, \Theta_{j}\right) p\left(\boldsymbol{\theta}_{\boldsymbol{j}} \mid \Theta_{j}\right) d \boldsymbol{\theta}_{\boldsymbol{j}} \\
& =\int_{\Theta_{j}} p\left(A_{\Theta_{k}}^{(e)} \mid \boldsymbol{\theta}_{\boldsymbol{j}}\right) p\left(\boldsymbol{\theta}_{\boldsymbol{j}} \mid \Theta_{j}\right) d \boldsymbol{\theta}_{\boldsymbol{j}} .
\end{aligned}
$$

The sampling distribution of $p\left(\theta_{j} \mid \Theta_{j}\right)$ can be assumed as a uniform distribution under each $\Theta_{j}$. Assuming $p\left(A_{\Theta_{k}}^{(e)} \mid \boldsymbol{\theta}_{\boldsymbol{j}}\right)$ is constant for each $j$ since the the quantization interval of $\Theta_{j}$ is small enough, $p\left(A_{\Theta_{k}}^{(e)} \mid \boldsymbol{\theta}_{\boldsymbol{j}}\right)$ is reduced to $p\left(A_{\Theta_{k}}^{(e)} \mid \boldsymbol{\theta}_{\boldsymbol{j}}^{*}\right)$, where $\boldsymbol{\theta}_{\boldsymbol{j}}^{*}$ is the mean value of the interval $\Theta_{j} \cdot p\left(A_{\Theta_{k}}^{(e)} \mid \boldsymbol{\theta}_{j}^{*}\right)$ is derived as follows from the definition of $f_{\text {dist }}$ in sec 3.1 and assuming that $f_{\text {dist }}$ obeys a gaussian

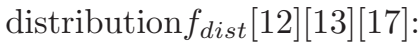

$$
p\left(A_{\Theta_{k}}^{(e)} \mid \boldsymbol{\theta}_{j}^{*}\right)=\alpha_{\boldsymbol{\theta}_{j}^{*}}^{(e)} \exp \left(-\left(d_{M}^{(e)}(k, j)\right)^{2}\right)
$$

where $I_{r}^{(e)}(\boldsymbol{\theta})$ is the edge image rendered from the posture $\boldsymbol{\theta}$, and

$$
d_{M}^{(e)}(k, j)=\frac{f_{\text {dist }}\left(A_{\Theta_{k}}^{(e)} ; I_{r}^{(e)}\left(\boldsymbol{\theta}_{j}^{*}\right)\right)}{\sigma_{M}^{(e)}} .
$$


$\sigma_{M}^{(e)^{2}}$ is the variance of the value of $f_{\text {dist }}\left(A_{\Theta_{k}}^{(e)} ; I_{r}^{(e)}\left(\boldsymbol{\theta}_{j}^{*}\right)\right) \cdot \sigma_{M}^{(e)}$ is experimentally determined. $\alpha_{\boldsymbol{\theta}_{j}^{*}}^{(e)}$ is normalization constant,

$$
\alpha_{\boldsymbol{\theta}_{j}^{*}}^{(e)}=\left(\sum_{k} \exp \left(-\left(d_{M}^{(e)}(k, j)\right)^{2}\right)\right)^{-1} .
$$

In the same manner as the above,

$$
p\left(A_{\Theta_{k}}^{(s)} \mid \boldsymbol{\theta}_{\boldsymbol{j}}^{*}\right)=\alpha_{\boldsymbol{\theta}_{j}^{*}}^{(s)} \exp \left(-\left(d_{M}^{(s)}(k, j)\right)^{2}\right)
$$

where $I_{r}^{(s)}(\boldsymbol{\theta})$ is the silhouette generated from $\boldsymbol{\theta}$, and

$$
d_{M}^{(s)}(k, j)=\frac{f_{s k i n}\left(A_{\Theta_{k}}^{(s)} ; I_{r}^{(s)}\left(\boldsymbol{\theta}_{j}^{*}\right)\right)}{\sigma_{M}^{(s)}}
$$

$\sigma_{M}^{(s)^{2}}$ is the variance of the value of $f_{s k i n}\left(A_{\Theta_{k}}^{(s)} ; I_{r}^{(s)}\left(\boldsymbol{\theta}_{\boldsymbol{j}}^{*}\right)\right) \cdot \alpha_{\boldsymbol{\theta}_{\boldsymbol{j}}^{*}}^{(s)}$ is normalization constant,

$$
\alpha_{\boldsymbol{\theta}_{j}^{*}}^{(s)}=\left(\sum_{k} \exp \left(-\left(d_{M}^{(s)}(k, j)\right)^{2}\right)\right)^{-1}
$$

\subsection{Likelihood of Appearance}

In this section, we explain the evaluation of the likelihood of an appearance $p\left(I^{(e)} \mid A_{\Theta_{k}}^{(e)}\right)$. The likelihood is defined based on the definition of $f_{\text {dist }}$ as

$$
p\left(I^{(e)} \mid A_{\Theta_{k}}^{(e)}\right)=\beta_{\Theta_{k}}^{(e)} \exp \left(-\frac{\left(f_{d i s t}\left(A_{\Theta_{k}}^{(e)} ; I^{(e)}\right)\right)^{2}}{\sigma_{I}^{(e)^{2}}}\right) .
$$

where, $\sigma_{I}^{(e)^{2}}$ is the variance of $\left(f_{\text {dist }}\left(A_{\Theta_{k}}^{(e)} ; I^{(e)}\right)\right)$. It is experimentally determined. The normalization constant $\beta_{\Theta_{k}}^{(e)}$ is derived from the integral condition of probabilistic distributions:

$$
\int p\left(i^{(e)} \mid A_{\Theta_{k}}^{(e)}\right) d i^{(e)}=1
$$

Assuming that $p\left(i^{(e)} \mid A_{\Theta_{k}}^{(e)}\right)$ can be large value only for $i^{(e)}$ of hand images and is 0 for most of other $i^{(e)}$,

$$
\begin{aligned}
\int p\left(i^{(e)} \mid A_{\Theta_{k}}^{(e)}\right) d i^{(e)} & \approx \int p\left(I_{r}^{(e)}\left(\theta_{l}\right) \mid A_{\Theta_{k}}^{(e)}\right) d \theta_{l} \\
& \approx \sum_{l} p\left(I_{r}^{(e)}\left(\theta_{l}^{*}\right) \mid A_{\Theta_{k}}^{(e)}\right) \cdot \delta \\
& =\beta_{\Theta_{k}}^{(e)} \sum_{l} \exp \left(-\left(d_{M}^{(e)}(k, l)\right)^{2}\right) \cdot \delta \\
& \equiv 1
\end{aligned}
$$

where $\delta$ is the range of the quantization of $\Theta$.

$$
\beta_{\Theta_{k}}^{(e)}=\left(\sum_{l} \exp \left(-\left(d_{M}^{(e)}(k, l)\right)^{2}\right) \cdot \delta\right)^{-1}
$$


When $A_{\Theta_{k}}^{(e)}$ wrongly matches to many of $I_{r}^{(e)}\left(\theta_{l}\right)(l \neq k), \beta_{\Theta_{k}}^{(e)}$ becomes small. On the other hand when a few of those, $\beta_{\Theta_{k}}^{(e)}$ becomes large. It means that ambiguous appearance model, which is easy to mis-match to other posture's appearances, are automatically low evaluated.

\section{Estimation of More Accurate Posture Parameters}

Posture parameters of the best-matched model are slightly different from that of the hand of an input image due to quantization errors of posture parameters. Thus, more accurate parameters must be estimated.

The wireframe CG model of the hand is deformed so that the model is matched to an input image, and the 3-D hand shape is reconstructed from the deformed model[19]. In this method, while the curved surface shape of the hand is reconstructed, posture parameters are not estimated. We deform the CG model so that the appearance of the model is matched to those of the input image by using this method. The accurate posture parameters are estimated from coordinates of the vertices of the triangle patches of the deformed wireframe model.

Parameters are estimated by the following steps of a procedure.

1. We make correspondences of edges of the best-matched model to those of the input image.

2. The change of the appearance is evaluated from the correspondences so that the edges of the model move toward those of the input image.

3. In order to reduce the huge search region of posture parameters due to the high DOF of human hand, available deformations of surface mesh of the CG model are learned by PCA in advance for each of typical postures, and then the best approximated mesh deformation is estimated by the projection to the PCA subspace.

4. Return to 1 . We make correspondences of edges of the CG model deformed at 3. to those of the input image. CG model is deformed by the change of appearance evaluated from the correspondence, again. Repeat these processes.

5. We evaluate the 2-dimensional Euclidean norm of the vertices of the triangle patches between the deformed CG model and CG model generated by the posture parameters. The sum of the norms is minimized using steepest descent method. The posture parameters with minimized sum of the norms are the posture estimate.

\section{Experiment}

We did the experiment of posture tracking for 250 hand images. The resolution of the images is $320 \times 240$. The images are captured by $30 \mathrm{fps}$. In the conventional method, where $p\left(I \mid \Theta_{j}\right)=p\left(I \mid A_{\Theta_{j}}^{(e)}\right) p\left(I \mid A_{\Theta_{j}}^{(s)}\right)$ is used as a matching criterion, $70.4 \%$ images are correctly matched. In our method, $82.0 \%$ images are correctly matched. The success rates show effectiveness of our method. 


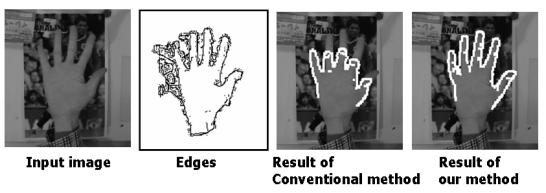

Fig. 7. Experimental result
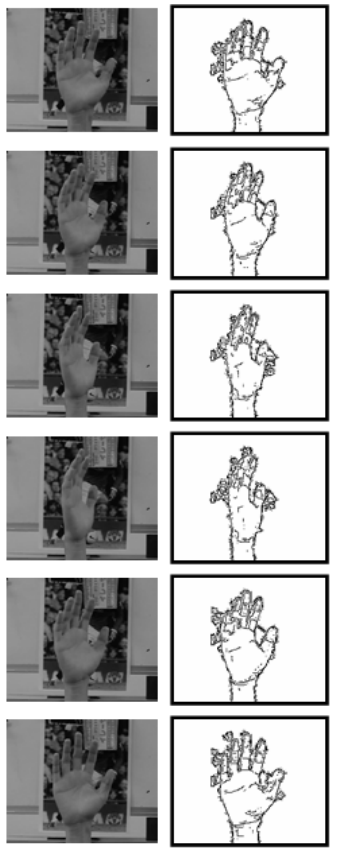

Input images

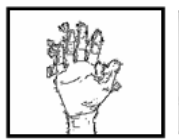

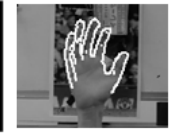
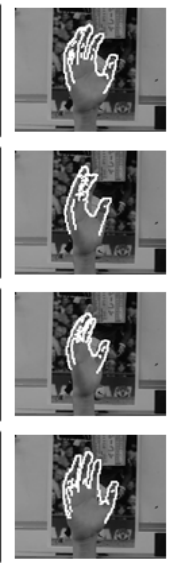

Edges Edges of estimation result
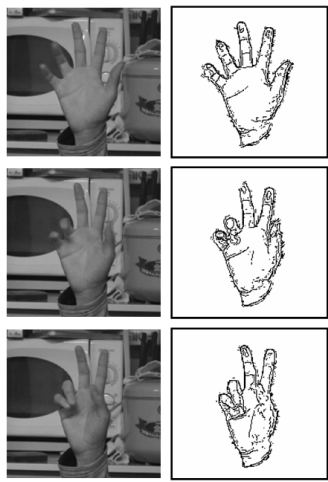

Input images

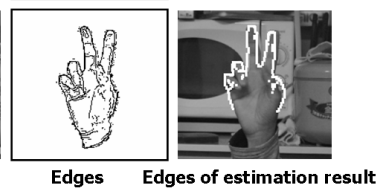

Fig. 9. Experimental result
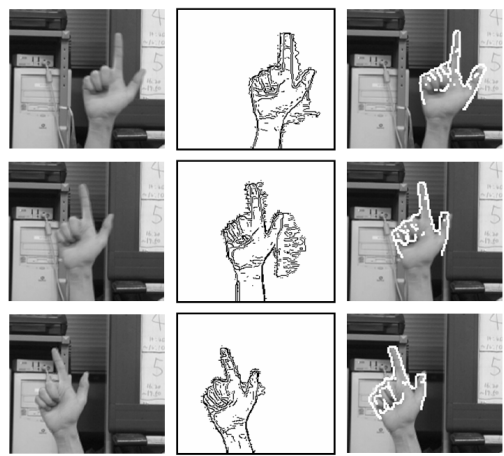

Input images
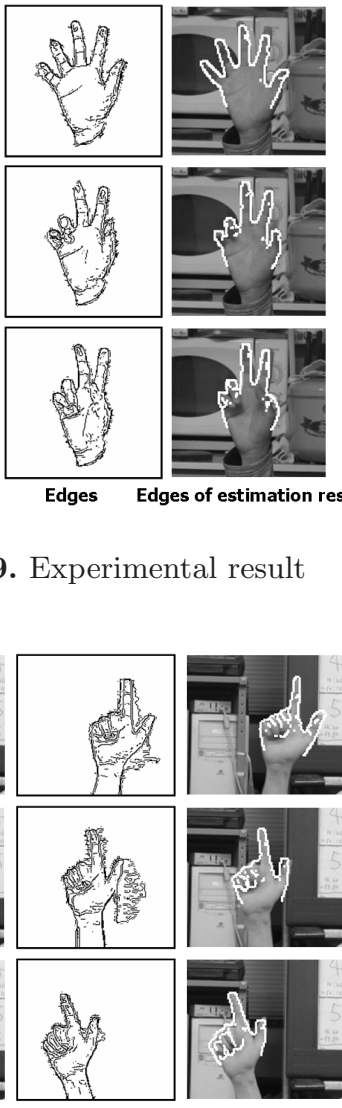

Edges Edges of estimation result

Fig. 8. Experimental result

Fig. 10. Experimental result

The example of the image which is correctly matched in our method while mis-matched in the conventional method, is shown in Fig. 7. While the wrong fist hand shape is matched in conventional method, the correct flat shape is matched in our method.

Fig. 8 shows the results of an image sequence. In the input images, fingers are partially occluded and the edges of the background are confusingly appeared near the fingers. Such cases causing mismatches are correctly matched in our method. Fig. 9 and Fig. 10 show the tracking results for other hand shapes. These images are also correctly matched in our method.

\section{Conclusion and Discussion}

The paper introduces a Bayesian form of evaluation of posture candidates for hand tracking in complex backgrounds. The novel concept of Mistakenly 
Matching Likelihood (MML) enables to discriminate the true posture candidate from other confusing ones when the mismatch of image features frequently occurs. Experimental results for tracking of the real human hand show the effectiveness of this evaluation method. Additional image features like optical flows or range other than edges and silhouette should be considered on this framework as future work.

\section{Acknowledgment}

This work is supported in part by Grant-in-Aid for Scientific Research from Ministry of Education, Science, Sports, and Culture, Japanese Government, No.15300058.

The 3-D model of the real human hand was provided by courtesy of Prof. F. Kishino and Prof. Y. Kitamura, Osaka University.

\section{References}

1. Liu, X., Fujimura, K.: Hand Gesture Recognition using Depth Data. In: Proc. 6th Int. Conf. on Automatic Face and Gesture Recognition, pp. 529-534 (2004)

2. Iwai, Y., Yagi, Y., Yachida, M.: Estimation of Hand Motion and Position from Monocular Image Sequence. In: Li, S., Teoh, E.K., Mital, D., Wang, H. (eds.) ACCV1995. LNCS, vol. 1035, pp. 230-234. Springer, Heidelberg (1996)

3. Lee, S.U., Cohen, I.: 3D Hand Reconstruction from a Monocular View. In: Proc. 17th Int. Conf. on Pattern Recognition, vol. 3, pp. 310-313 (1995)

4. Kameda, Y., Minoh, M., Ikeda, K.: Three Dimensional Pose Estimation of an Articulated Object from its Silhouette Image. In: ACCV 1993, pp. 612-615 (1993)

5. Shimada, N., Kimura, K., Shirai, Y.: Real-time 3-D Hand Posture Estimation based on 2-D Appearance Retrieval Using Monocular Camera. In: Proc. Int. Workshop on RATFG-RTS, pp. 23-30 (2001)

6. Imai, A., Shimada, N., Shirai, Y.: 3-D Hand Posture Recognition by Training Contour Variation. In: Proc. of The 6th Int. Conf. on Automatic Face and Gesture Recognition, pp. 895-900 (2004)

7. Cootes, T.F., Taylor, C.J., Cooper, D.H., Graham, J.: Active Shape Models-Their Training and Application. COMPUTER VISION AND IMAGE UNDERSTANDING 61(1), 38-59 (1995)

8. Jeong, M., Kuno, Y., Shimada, N., Shirai, Y.: Recognition of shape-changing hand gestures. IEICE Trans. inf.Syst. E85-D(10), 1678-1687 (2002)

9. Isard, M., Blake, A.: Visual tracking by stochastic propagation of conditional density. In: Proc. European Conf. Computer Vision, pp. 343-356 (1996)

10. Isard, M., Blake, A.: ICONDENSATION:Unifying low-level and high-level tracking in a stochastic framework. In: Proc. European Conf. Computer Vision, pp. 767-781 (1996)

11. Heap, T., Hogg, D.: Wormholes in Shape Space:Tracking through Discontinuous Changes in Shape. In: 6th Int. Conf. on Computer Vision, pp. 344-349 (1998)

12. Zhou, H., Huand, T.S.: Tracking Articulated Hand Motion with Eigen Dynamics Analysis. 9th Int. Conf. on Computer Vision 2, 1102-1109 (2003) 
13. Stenger, B., Thayananthan, A., Torr, P.H.S., Cipolla, R.: Model-Based Hand Tracking Using a Hierarchical Bayesian Filter. IEEE Transactions on Pattern Analysis and Machine Intelligence 28(9), 1372-1384 (2006)

14. Wu, Y., Lin, J., Huang, T.S.: Analyzing and Capturing Articulated Hand Motion in Image Sequences. IEEE TRANS. ON PATTERN ANALYSIS AND MACHINE INTELLIGENCE 27(12), 1910-1922 (2005)

15. Athitsos, V., Sclaroff, S.: Estimating 3D Hand Pose from a Cluttered Image. In: IEEE Conf. on Computer Vision and Pattern Recognition, vol. II, pp. 432-439. IEEE Computer Society Press, Los Alamitos (2003)

16. Barrow, H.G., Tenenbaum, J.M., Bolles, R.C., Wolf, H.C.: Parametric correspondence and chamfer matching: Two new techniques for image matching. In: Proc. 5th Int. Joint Conf. Artificial Intelligence, pp. 659-663 (1977)

17. Blake, A., Isard, M.: Active Contours. Springer, Heidelberg (1998)

18. Navaratnam, R., Thayananthan, A., Torr, P.H.S., Cipolla, R.: Hierarchical PartBased Human Body Pose Estimation. In: Proc. British machine Vision Conference (2005)

19. Heap, T., Hogg, D.: Towards 3D Hand Tracking using a Deformable Model. In: 2nd Int. Conf. on Automatic Face and Gesture Recognition, pp. 140-145 (1996) 